\title{
Verhaltenstherapie - Totgesagte leben länger
}

\author{
Michael Linden \\ Forschungsgruppe Psychosomatische Rehabilitation an der Charité Universitätsmedizin Berlin und Abteilung für psychische und \\ psychosomatische Erkrankungen am Rehabilitationszentrum Seehof der Deutschen Rentenversicherung Bund, Teltow/Berlin, Deutschland
}

Diese Ausgabe der Verhaltenstherapie enthält einen Artikel von Frau Dr. Ulrike Kübler zum Thema «Verhaltensanalyse in der Verhaltenstherapie». Damit wird eine Serie unter der Überschrift «Konturen der Verhaltenstherapie» eröffnet, in der Kernelemente der Verhaltenstherapie beschrieben werden. Diese Zeitschrift heißt Verhaltenstherapie und versteht sich als deutschsprachige wissenschaftliche und klinische Fachzeitschrift, die das Verfahren «Verhaltenstherapie», so wie es in den Psychotherapierichtlinien genannt wird, zum Thema hat. Es ist von daher die Aufgabe dieser Zeitschrift, in Fachbeiträgen darzulegen, was unter Verhaltenstherapie zu verstehen ist und gegebenenfalls auch, was nicht.

Diese Aufgabe ist derzeit dringender den je, da es sehr vielfältige Diskussionen darüber gibt, ob es die bisherigen Psychotherapieverfahren oder -schulen weiterhin geben sollte. Als Alternativkonzepte werden die allgemeine Psychotherapie, psychologische Psychotherapie, ganzheitliche Psychotherapie, störungsspezifische Psychotherapie usw. vorgeschlagen. Die Herausgeber der VeRHALTENSTHERAPIE vertreten in dieser Fachdiskussion die Position, dass eine Unterscheidung zwischen verschiedenen Psychotherapieverfahren möglich und sinnvoll, wenn nicht sogar geboten ist und dass sich Verhaltenstherapie von anderen Therapieverfahren abgrenzen lässt.

Die Vertreter der sogenannten störungsspezifischen Psychotherapie übersehen, dass die meisten störungsadaptierten Therapieinterventionen nur vor dem Hintergrund einzelner Psychotherapieverfahren zu verstehen und anzuwenden sind. Um störungsspezifische Psychotherapien durchführen zu können, werden psychotherapeutische Grundkompetenzen benötigt, um das, was störungsspezifisch gefordert wird, überhaupt umsetzen zu können. Wer nicht weiß, was ein «kognitives Schema» ist und es mit «ich denke mir was» verwechselt; wer keine automatischen Gedanken erkennen und beschreiben kann oder nichts von den Methoden der kognitiven Verhaltenstherapie versteht, durch die sich Kognitionen modifizieren lassen, wird keine fachlich überzeugende Änderung von kognitiven Schemata bewirken können. Die klinische Erfahrung zeigt, dass dann eher mit Nebenwirkungen gerechnet werden muss.

Damit ist auf einen zweiten Punkt hinzuweisen. Psychotherapieverfahren sind «Schulen», d.h. Theorien und Psychotherapiekompetenzen, in denen Psychotherapeuten ausgebildet werden. Eine Ausbildung in störungsspezifischer Psychotherapie ist grundsätzlich nicht möglich, da eine Ausbildung in Psychotherapie maximal 3 Jahre umfasst, in denen nur wenige Fälle behandelt werden können. Die Zahl der psychischen Störungen geht jedoch in die Hunderte. Wollte man tatsächlich das Konzept einer störungsspezifischen Psychotherapieausbildung ernst nehmen, dann würde dies bedeuten, dass am Ende der Psychotherapieaus- oder Weiterbildung ein Therapeut maximal 3 Krankheiten behandeln könnte. Es kann also gar keine störungsspezifische Psychotherapieausbildung geben, sondern nur eine Ausbildung in einem Psychotherapieverfahren, das ermöglicht, sehr unterschiedliche Krankheitsbilder störungsspezifisch zu behandeln.

Damit stellt sich als nächste Frage, ob es eine allgemeine Grundausbildung in Psychotherapie gibt. Bei entsprechenden Diskussionen wird immer wieder vorgetragen, dass letztlich doch alle Psychotherapeuten dasselbe machen würden. Bei genauerer Betrachtung handelt es sich bei diesen Argumenten jedoch in der Regel um eine fehlende Präzision in der Unterscheidung von Therapiezielen und Therapieinterventionen. Mit allen Therapien soll erreicht werden, dass die vorliegenden Beschwerden abnehmen, dass ein Mensch sich in seiner Persönlichkeit kongruent weiterentwickelt, oder dass sich im Verlauf einer Therapie das Verhalten von Patienten ändert. Therapieverfahren können auch nicht dadurch unterschieden werden, dass man auf grundlagenwissenschaftliche Kenntnisse aus der Psychologie oder Neurobiologie rekurriert. Selbstverständlich sollte eine Gesprächspsychotherapie, Verhaltenstherapie oder psychodynamische Psychotherapie gleichermaßen in der Behandlung einer Angsterkrankung

\section{KARGER \\ Fax +497614520714}

Information@Karger.com

www.karger.com (c) 2013 S. Karger GmbH, Freiburg

$1016-6262 / 13 / 0232-0072 \$ 38.00 / 0$

Accessible online at:

www.karger.com/ver
Prof. Dr. Michael Linden

Rehabilitationszentrum Seehof der Deutschen Rentenversicherung Bund

Lichterfelder Allee 55, 14513 Berlin, Deutschland

michael.linden@charite.de 
neurobiologisch die neuronalen inhibitorischen Rückkopplungsschleifen vom Frontalhirn zur Amygdala stärken oder psychologisch die individuellen Weltbewertungen verändern. Der Unterschied zwischen verschiedenen Psychotherapieverfahren ist die Methodik, mit der die jeweiligen identischen Ziele erreicht werden. Verhaltenstherapeuten machen Hausaufgaben oder Expositionsübungen, was nicht in das Repertoire psychodynamischer Psychotherapeuten oder Gesprächspsychotherapeuten gehört. Ebenso wenig gehört eine Übertragung und Triangulation in das Repertoire eines Verhaltenstherapeuten. Man kann auch nicht beides gleichzeitig abdecken und es ist zu hoffen, dass man das eine oder das andere richtig macht, anstatt beides falsch und nebenwirkungsträchtig durchzuführen. Die Erfahrungen in der täglichen Psychotherapieausbildung zeigen, dass es in der kurzen Ausund -weiterbildungszeit von 3 Jahren gerade einmal gelingt, grundsätzliche Kompetenzen, Sprachstile, Fragestile und theoretische Modelle einer Therapieschule zu vermitteln. Wer regelmäßig Therapiebänder anhört, in denen sich junge Therapeuten redlich bemühen, etwas Richtiges zu sagen, weiß, dass dies ein langwieriger Lernprozess ist. Ebenso wenig wie es Sinn macht, 100 Stunden Musikunterricht auf das Erlernen von Geige, Querflöte, Klavier, Trompete und Triangel zu verteilen, macht es Sinn, in der Psychotherapeutenaus- und -weiterbildung die Kollegen mit sehr unterschiedlichen theoretischen Konzepten zu konfrontieren, was regelhaft zur Verwirrung führt. Die Kollegen benutzen dann Fachbegriffe, ohne zu verstehen, was damit gemeint ist und halten sich im schlimmsten Fall noch für klug.

Ein weiteres, immer wieder vorgebrachtes Argument gegen eine Differenzierung von Schulen ist, dass die eben angesprochenen Basistechniken und Kompetenzen gar nicht wichtig seien, weil der gesamte Therapieeffekt letztlich über die therapeutische Beziehung zu erklären sei. Dafür gibt es in der wissenschaftlichen Literatur keinerlei Evidenz. Selbst die Gesprächspsychotherapie oder die mit ihr verwandte sogenannte allgemeine Psychotherapie, die diesem Aspekt seit jeher besondere Aufmerksamkeit widmen, kommen damit nicht aus, wie deren Überprüfung durch den wissenschaftlichen Beirat Psychotherapie und den gemeinsamen Bundesausschuss gezeigt haben. Es ist auch nicht richtig, dass jede Therapie zum gleichen Ergebnis führt, selbst wenn dies gelegentlich in Abstracts international publizierter Artikel behauptet wird, deren Daten dann jedoch belegen, dass unterschiedliches Vorgehen durchaus unterschiedliche Effekte hat.

Zusammenfassend gibt es bei dem derzeitigen Stand der wissenschaftlichen Forschung, Konzeptbildung und Ausbildungsrahmenbedingungen also gute Gründe, weiterhin von Verhaltenstherapie in Abgrenzung zu psychodynamischer Psychotherapie, klientenzentrierter Psychotherapie, Gestaltpsychotherapie usw. zu sprechen. Es gibt hinreichende empirische Evidenz dafür, dass die Verhaltenstherapie ein Psychotherapieverfahren ist, das ein hinreichend differenziertes psychotherapeutisches Handwerkszeug zur Verfügung stellt, um ein breites Spektrum unterschiedlichster psychischer Störungen mit unterschiedlichem Komplexitätsgrad adäquat behandeln zu können. Die verhaltenstherapeutischen Techniken und Methoden sind auch hinreichend operationalisiert, um erkennen zu können, wenn ein Behandler von den Regeln der Kunst abweicht oder sogar einen Kunstfehler begeht. Dabei sind sich die Herausgeber der Verhaltenstherapie mit Vertretern anderer Psychotherapieverfahren durchaus einig, dass nicht alles Verhaltenstherapie ist, was wirkt oder auf grundlagenwissenschaftliche Befunde Bezug nimmt. Zusammengefasst erfüllt die Verhaltenstherapie damit die Voraussetzungen, um als Verfahren der Krankenbehandlung in einem Sozialversicherungssystem gleichrangig neben anderen Behandlungsalternativen eingesetzt werden zu können. 